\title{
The effects of cues on neurons in the basal ganglia in Parkinson's disease
}

\author{
Sridevi V. Sarma ${ }^{1 *}{ }^{\dagger}$, Ming L. Cheng ${ }^{2 \dagger}$, Uri Eden $^{3}$, Ziv Williams $^{4}$, Emery N. Brown $^{5,6}$ and \\ Emad Eskandar ${ }^{4}$ \\ 1 Biomedical Engineering Department, Institute for Computational Medicine, Johns Hopkins University, Baltimore, MD, USA \\ 2 Department of Neurosurgery, Center for Neurorestoration, Alpert Medical School at Brown University, Providence, RI, USA \\ ${ }^{3}$ Department of Mathematics and Statistics, Boston University, Boston, MA, USA \\ ${ }^{4}$ Department of Neurosurgery, Al-Rohdan Laboratories - MGH-HMS Center for Nervous System Repair, Massachusetts General Hospital, Boston, MA, USA \\ ${ }^{5}$ Division of Health Sciences and Technology, Harvard Medical School/Massachusetts Institute of Technology, Cambridge, MA, USA \\ ${ }^{6}$ Department of Brain and Cognitive Sciences, Massachusetts Institute of Technology, Cambridge, MA, USA
}

\section{Edited by:}

John T. Gale, Cleveland Clinic, USA

Reviewed by:

Richardson N. Leão, Karolinska

Institute, Sweden

Andre Machado, Cleveland Clinic, USA

\section{*Correspondence:}

Sridevi V. Sarma, Biomedical

Engineering Department, Institute

for Computational Medicine, Johns

Hopkins University, 3400 North

Charles Street, 316c Hackerman

Hall, Baltimore, MD 21218, USA.

e-mail: sree@jhu.edu

${ }^{\dagger}$ These authors contributed equally to this work.
Visual cues open a unique window to the understanding of Parkinson's disease (PD). These cues can temporarily but dramatically improve PD motor symptoms. Although details are unclear, cues are believed to suppress pathological basal ganglia (BG) activity through activation of corticostriatal pathways. In this study, we investigated human BG neurophysiology under different cued conditions. We evaluated bursting, $10-30 \mathrm{~Hz}$ oscillations (OSCs), and directional tuning (DT) dynamics in the subthalamic nucleus (STN) activity while seven patients executed a two-step motor task. In the first step (predicted +cue), the patient moved to a target when prompted by a visual go cue that appeared $100 \%$ of the time. Here, the timing of the cue is predictable and the cue serves an external trigger to execute a motor plan. In the second step, the cue appeared randomly $50 \%$ of the time, and the patient had to move to the same target as in the first step. When it appeared (unpredicted $+\mathrm{cue}$ ), the motor plan was to be triggered by the cue, but its timing was not predictable. When the cue failed to appear (unpredicted-cue), the motor plan was triggered by the absence of the visual cue. We found that during predicted +cue and unpredicted - cue trials, OSCs significantly decreased and DT significantly increased above baseline, though these modulations occurred an average of $640 \mathrm{~ms}$ later in unpredicted -cue trials. Movement and reaction times were comparable in these trials. During unpredicted +cue trials, OSCs, and DT failed to modulate though bursting significantly decreased after movement. Correspondingly, movement performance deteriorated. These findings suggest that during motor planning either a predictably timed external cue or an internally generated cue (generated by the absence of a cue) trigger the execution of a motor plan in premotor cortex, whose increased activation then suppresses pathological activity in STN through direct pathways, leading to motor facilitation in PD.

Keywords: neuron, neuropathology, Parkinson disease, neuromodulation, cueing

\section{INTRODUCTION}

An estimated 6.5 million people world-wide have Parkinson disease (PD), a chronic progressive neurological disorder that occurs when dopaminergic neurons in the midbrain degenerate, causing motor deficits including tremor, rigidity, and bradykinesia (Lang and Lozano, 1998a,b). An intriguing aspect of PD is the dynamic nature of these motor symptoms. Clinical observations show that tremor is attenuated with movement (Lang and Lozano, 1998b) while visual and auditory cues improve gait (Georgiou et al., 1993; Morris et al., 1996; Azulay et al., 1996, 1999; Suteerawattananon, 2004), movement velocity, movement accuracy (Majsak et al., 1998), reaction times (Kühn et al., 2004), and off freezing (Kompoliti et al., 2000). There are different proposals regarding the mechanism underlying cue-related improvements in motor function. One hypothesis is that alternative preserved visual-motor pathways bypassing the basal ganglia
(BG) facilitate motion responsiveness to visual cues (Glickstein and Stein, 1991). Another hypothesis is that increased cortical drive associated with cues leads to transient dampening of pathological $10-30 \mathrm{~Hz}$ oscillations in the BG, which in turn facilitates movement (Amirnovin et al., 2004).

Previous independent works suggest that in PD patients, (1) cues lead to increased cortical activity in premotor and motor areas which facilitates movement and (2) cues lead to suppression of pathological activity in the subthalamic nucleus (STN) of the BG which facilitates movement. Jahanshahi et al. (1995) showed increased cortical activity (when compared to rest) in cortical regions when PD patients either self-initiated movements or moved in response to a tone presented at a predicted rate. Kühn et al. (2004) showed that pathological $10-30 \mathrm{~Hz}$ oscillations (OSCs) in local field potentials from the STN region of PD patients decreased immediately after a cue to move was 
presented, with an onset latency that strongly correlated with mean reaction times. These results are consistent with findings that in PD patients, beta oscillations in single-unit recordings of STN neurons were suppressed during visually guided movement (Amirnovin et al., 2004) and active voluntary movement (Levy et al., 2002). Taken together, the literature may suggest that in PD patients cue-driven cortical activity is responsible for decreasing pathological BG activity that facilitates motor function.

It is important that the cortical activation reported in (Jahanshahi et al., 1995) occurred when patients could either predict the timing of the cue or when they self-initiated movements. Two important questions that we ask here are (1) "what happens when the timing of cues cannot be predicted by patients?" and (2) "what if an expected cue never appears forcing the patient to move in the absence of a cue?" In the same study, Jahanshahi showed that when patients could not predict cue timing, reaction times increased and there was no significant increase in cortical activity.

We set out to answer these two questions with respect to BG neurophysiology and movement-related behavior in PD patients. Our hypothesis was that the inability to predict cue timing would diminish dampening of pathological BG activity observed when cues are presented in a predictable fashion. We compared the effects of predictable and unpredictable cues on behavior and spiking activity in 28 STN neurons recorded from seven PD patients executing a two-step center-out task during deep brain stimulation surgery.

\section{MATERIALS AND METHODS SUBJECTS}

Seven patients undergoing deep brain stimulator placement for the treatment of PD were included in the study. All patients had idiopathic PD with a Hoehn-Yahr score of three or higher and had a documented response to L-dopa replacement therapy. All patients received a thorough pre-operative neurological exam. Exclusion criteria for surgery included those patients with Parkinson "plus" syndromes, cognitive impairment, active psychiatric disorders, or anatomic abnormalities on magnetic resonance imaging (MRI) (Amirnovin et al., 2006). None of the patients had undergone prior surgery for the treatment of PD. Informed consent for the study was obtained in strict accordance with a protocol approved by the Institutional Review Board and the multidisciplinary movement disorders assessment committee at the Massachusetts General Hospital. The decision to offer surgery was based on clinical indications alone, and bore no relation to the patients' participation in this study. To ensure that the patients were comfortable with performing the behavioral joystick task, they practiced the task prior to the surgery until they reached $90 \%$ success or more on all trial types. Subjects were able to remove their hand from the joystick or stop the task at any time. At all-time points before and during surgery, the patients had the clear understanding that their participation was not related to the surgical outcome, and that they could withdraw from the study at any time.

\section{ELECTROPHYSIOLOGY}

Anti-Parkinsonian medications were withheld the night before surgery. No sedatives were given prior to or during performance of recordings. A local anesthetic was used prior to the incision and burr hole placement. The stereotactic localization using pre-operative MRI and computerized tomography, as well as general techniques of intraoperative microelectrode recordings have been described previously (Abosch et al., 2002; Amirnovin et al., 2006). Single-unit recordings were made from the dorsal-lateral motor sub-territory of the STN based on stereotactic localization and reconstructions of the electrode trajectories (Abosch et al., 2002). The STN has characteristic high firing rates in comparison to the surrounding structures (Hutchison et al., 1998) and has clear dorsal and ventral borders that are evident when reconstructing neuronal activity along the electrode trajectories. Once within the STN, no attempt was made to explicitly select cells based on presence or absence of movement-related activity, or on whether the cells responded to passive and/or volitional movement. This was done specifically to limit the potential for a sampling bias.

We used an array of 3 tungsten microelectrodes, separated by $2 \mathrm{~mm}$ and placed in a parasagittal orientation. The electrodes were advanced simultaneously in $50-\mu$ increments using a motorized micro-drive (Alpha Omega; Nazareth, Israel). The behavioral paradigm was controlled by a Macintosh G4 computer using custom-made software. Neuronal activity was bandpass filtered $(300 \mathrm{~Hz}-6 \mathrm{kHz})$ and sampled at $20 \mathrm{kHz}$. Spikes were sorted off-line using a standardized template-matching algorithm (Cambridge Electronics Design, Cambridge, England).

\section{BEHAVIORAL TASK}

Once the microelectrodes were in the STN and stable single units were obtained, the subjects viewed a computer monitor and performed a two-step behavioral task by moving a joystick (mounted such that movements were in a horizontal orientation with the elbow flexed at approximately $45^{\circ}$ ) with the contra-lateral hand. Each task pair consisted of a predictable cue trial followed by an unpredictable trial. Refer to Figure 1. In the predictable cue trial, a central fixation spot $\left(0.2^{\circ}\right.$ in diameter $)$ was first displayed for $500 \mathrm{~ms}$, after which, an array of four gray equally spaced circular targets ( $1^{\circ}$ in diameter) would appear in the "up", "right", "down", and "left" directions. After a variable delay interval ranging 500-1000 ms, one of the gray targets would turn green. Following another variable delay interval ranging from 500 to $1000 \mathrm{~ms}$, the central fixation spot would turn green indicating that the patients could move the joystick. Once within the target, patients were required to hold the cursor stationary for another $100 \mathrm{~ms}$. The stimuli on the screen would then erase, and the patients would be allowed to return the spring-loaded joystick to its resting position. It is important to note that patients knew a priori that a go cue will appear during these trials within a predictable window of time.

After completion of the first-step trial, the screen remained blank for $1000 \mathrm{~ms}$. This would be followed by one of two possible unpredictable trials. As before, a fixation spot and gray circular array would appear, but would be followed by a go cue with $50 \%$ chance. In these trials, the patients were required to move the joystick in the same direction as in the preceding trial. If the patient moved and a go cue appeared afterwards or if the patient waited too long before moving ( $\geq 4 \mathrm{~s}$ after fixation), he/she would not 


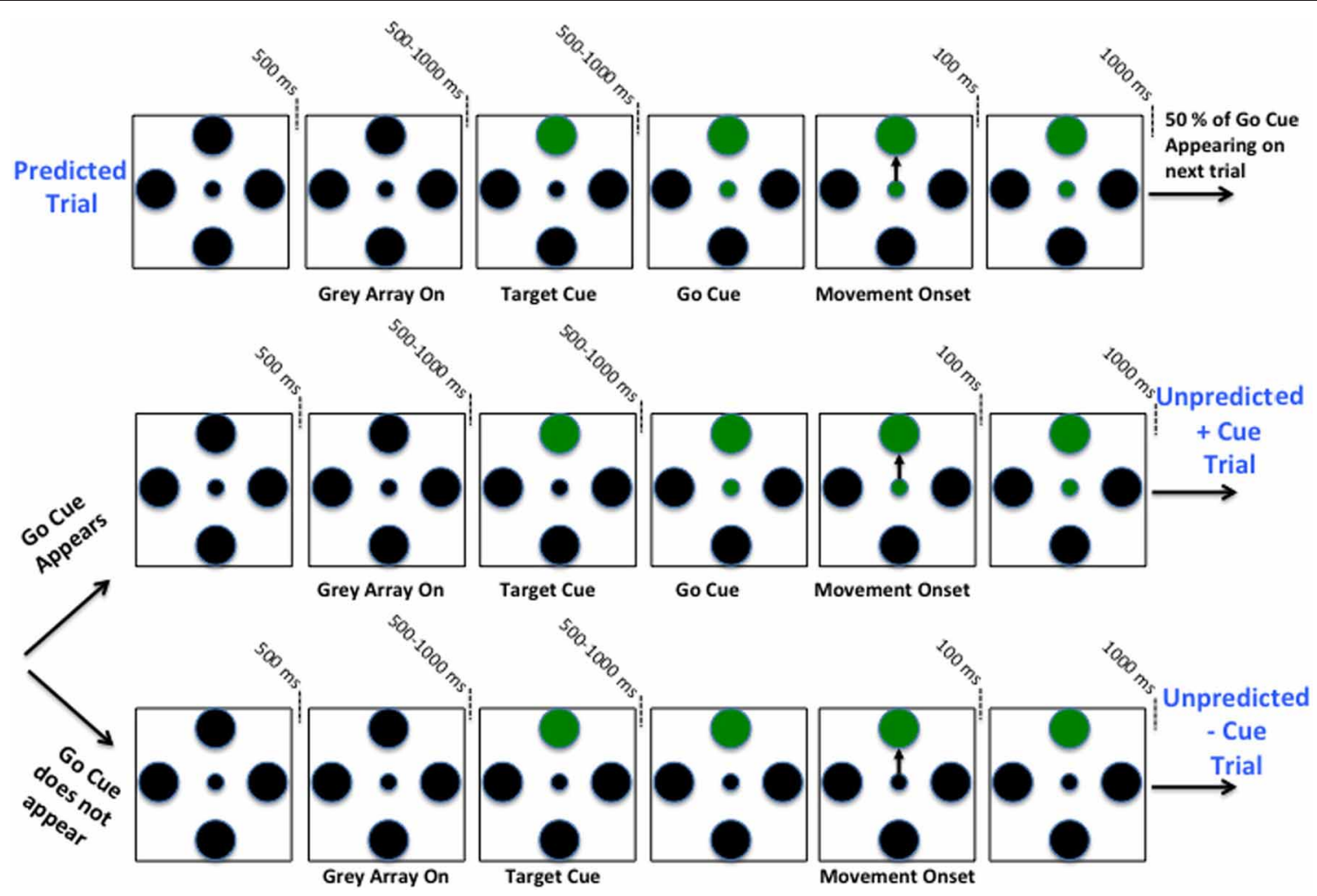

FIGURE 1 | Schematic of two-step sequential motor task. Predicted +Cue trial (Top), Unpredicted +Cue trial (middle), Unpredicted -Cue trial (bottom).

complete the second-step trial successfully. Therefore, patients had an incentive to wait for a possible go cue and if no go cue appeared within a short time period then the lack of the cue triggered movement. In fact, after $1 \mathrm{~s}$ following the presentation of a target cue, if the go cue does not appear, then with certainty it will not be coming and subjects should self-initiate the movement. Patients had to learn this over time, and when each trial was completed, a sound tone was generated indicating whether or not they completed it correctly.

It is important to note that the predictable trials and the unpredictable trials in which the go cue is presented are identical, with the exception that the timing of the go-cue in the latter is not as predictable. The trials were pseudo-randomly interleaved in blocks such that each direction and trial type was presented once within each block, rendering a 50\% chance of a go cue appearing on any given unpredicted trial. All directions and trial types were counterbalanced such that an equal number of directions and trials types were tested for each cell. Furthermore, variable delays for cue presentation on + cue trials were each timed separately. If patients strayed beyond the confines of a $60^{\circ}$-wide invisible corridor, moved the cursor to an incorrect target, failed to return the joystick to its resting position or failed to reach the target within $4 \mathrm{~s}$ from fixation, the trial would abort and repeat again. The patients were instructed to maintain their gaze on the center of the screen at all time-points during the trial. See Figure 1 for a schematic of two-step sequential motor task.
Table 1 below itemizes the distribution of trials and neurons recorded per patient that contributed to the models used for our analysis. As described below, the neurons used in the analysis gave rise to point process models that met a goodness-of-fit criterion.

\section{STATISTICAL ANALYSIS: POINT PROCESS MODELS OF STN DYNAMICS}

We analyze neuronal spiking activity in STN neurons by constructing point process models (Barbieri et al., 2001; Brown et al., 2001a,b; Truccolo et al., 2005, 2010). The point process framework has proven in practice to be a powerful and flexible framework that is capable of modeling spike train activity from a diverse range of neuronal types and neural circuits, such as: place cells from the rat hippocampus (Barbieri et al., 2001); retinal ganglion cells of the salamander, rabbit, and cat (Keat et al., 2001); neurons from the supplementary eye field of the macaque monkey (Kass and Ventura, 2001); and STN neurons of PD patients (Levy et al., 2001; Paninski, 2004; Eden et al., 2007; Czanner et al., 2008; Montgomery, 2008; Zelnikera et al., 2008; Sarma et al., 2010).

A point process model of a single neuron can capture the relative contribution of short and long-term history effects (temporal dependencies), movement direction, and the impact of external cues on the probability that the neuron will spike at any given time. Since STN neurons in PD patients exhibit pathological oscillations (Hutchison et al., 1994; Bergman et al., 1994; 
Table 1 | Distribution of trials and recorded neurons per patient.

\begin{tabular}{llllll}
\hline Patient ID & $\begin{array}{l}\text { Number of 2-step } \\
\text { paired trials } \\
\text { executed }\end{array}$ & $\begin{array}{l}\text { Total number of } \\
\text { neurons recorded }\end{array}$ & $\begin{array}{l}\text { Number of neurons } \\
\text { included in analysis } \\
\text { (Anticipated-cued } \\
\text { trials) }\end{array}$ & $\begin{array}{l}\text { Number of neurons } \\
\text { included in analysis } \\
\text { (Un-anticipated-cued-trials, } \\
\text { visually-guided) }\end{array}$ & $\begin{array}{l}\text { Number of neurons } \\
\text { included in analysis } \\
\text { (Un-anticipated-cued-trials, } \\
\text { self-initiated) }\end{array}$ \\
\hline 1 & 159 & 5 & 4 & 4 & 4 \\
2 & 38 & 3 & 3 & 3 & 1 \\
3 & 141 & 6 & 6 & 6 & 6 \\
4 & 24 & 7 & 2 & 2 & 2 \\
5 & 44 & 4 & 2 & 2 & 2 \\
6 & 189 & 7 & 7 & 4 & 2 \\
7 & 100 & 5 & 4 & 4 & 4 \\
\hline Total & 695 & 37 & 28 & 25 & 21 \\
\hline
\end{tabular}

Levy et al., 2000; Brown et al., 2001a,b; Dostrovsky and Bergman, 2004; Gale et al., 2009; Sarma et al., 2010 and more), the short and long-term history effects become significant factors on spiking probabilities. In addition, PD STN neurons exhibit increased directional tuning (DT) after movement (Crutcher and DeLong, 1984; Williams et al., 2005; Sarma et al., 2010), therefore movement direction will influence the models. Finally, since external cues such as visual cues and movement onset play a significant role in altering behavior in PD patients, it is likely that these extrinsic factors will also impact neuronal spiking probabilities of STN neurons. The point process framework thus enables us to study the dynamics of all characteristics (bursting, oscillations, and DT) in STN spiking activity simultaneously in an efficient and statistically sound manner.

A point process is a series of $0-1$ random events that occur in continuous time. For a neural spike train, the $1 \mathrm{~s}$ is individual spike times and the $0 \mathrm{~s}$ are the times at which no spikes occur. To define a point process model of neural spiking activity, in this analysis we consider an observation interval $(0, T]$, and let $N(t)$ be the number of spikes counted in interval $(0, T]$ for $t \in(0, T]$. A point process model of a neural spike train can be completely characterized by its conditional intensity function (CIF), $\lambda\left(t \mid H_{t}\right)$, defined as

$$
\lambda\left(t \mid H_{t}\right)=\lim _{\Delta \rightarrow 0} \frac{\operatorname{Pr}\left(N(t+\Delta)-N(t)=1 \mid H_{t}\right)}{\Delta}
$$

where $H_{t}$ denotes the history of spikes up to time $t$. It follows from (1) that the probability of a single spike in a small interval $(t, t+\Delta]$ is approximately

$$
\operatorname{Pr}\left(\text { spike in }(t, t+\Delta] \mid H_{t}\right) \cong \lambda\left(t \mid H_{t}\right) \Delta \text {. }
$$

When $\Delta$ is small, Equation (2) is roughly the spiking propensity at any time $t$ (Daley and Vere-Jones, 2003; Snyder and Miller, 1991). The well-known homogeneous Poisson process is a special point process in which all events are independent and the CIF does not dependent on history. Because the CIF characterizes a point process in its entirety, defining a model for a CIF defines a model for the spike train.
We use generalized linear models (GLMs; Truccolo et al., 2005) to characterize the CIF for each neuron. In a GLM, the log of the CIF is a modeled as a linear function of parameters that multiply the covariates which describe the neural activity dependencies The GLM is an extension of the multiple linear regression model in which the variable being predicted, in this case spike times, need not be Gaussian (McCullagh and Nelder, 1989). GLM also provides an efficient computational scheme for model parameter estimation and a likelihood framework for conducting statistical inferences based on the estimated model (Brown et al., 2003).

For each trial type, we define the CIF for each neuron to be a function of movement direction $d \in\{1,2,3,4\}$ which corresponds to $\{\mathrm{Up}$, Right, Left, and Down $\}$ and the neuron's spiking history in the preceding $150 \mathrm{~ms}$. Rather than estimating the CIF continuously throughout the entire trial, we estimate it over specific time windows around key epochs and at discrete time intervals each $1 \mathrm{~ms}$ in duration. In particular, we estimate the CIF over $500 \mathrm{~ms}$ during fixation (FX) and over $250 \mathrm{~ms}$ windows centered at the target cue onset (TC), go cue onset (GC), and movement onset (MV) onsets. Figure 2 highlights all of the time periods for which we estimate the CIF for each neuron.

We omit the superscripts denoting the epoch for a simpler read and define the rate function as

$$
\lambda\left(t \mid H_{t}, \theta\right)=\lambda^{s}\left(t \mid H_{t}, \theta\right) \cdot \lambda^{H}\left(t \mid H_{t}, \theta\right)
$$

where the component $\lambda^{s}\left(t \mid H_{t}, \theta\right)$ describes the effect of the behavioral stimulus (movement direction) on the neural response and the component $\lambda^{H}\left(t \mid H_{t}, \theta\right)$ describes the effect of spiking history on the neural response. $\theta=\{\alpha, \beta, \gamma\}$ is a parameter vector to be estimated from data and is defined below. The units of $\lambda\left(t \mid H_{t}, \theta\right)$ and $\lambda^{s}\left(t \mid H_{t}, \theta\right)$ are in spikes per second and $\lambda^{H}\left(t \mid H_{t}, \theta\right)$ is dimensionless. The idea to express the CIF as a product of a stimulus component and a temporal or spike history component was first suggested by (Kass and Ventura, 2001) and is appealing as it allows one to assess how much each component contributes to the spiking propensity of the neuron. If spiking history is not a factor associated with neural response, then $\lambda^{H}\left(t \mid H_{t}, \theta\right)$ will be very close to 1 for all times and Equation (3) becomes an inhomogeneous Poisson process. 


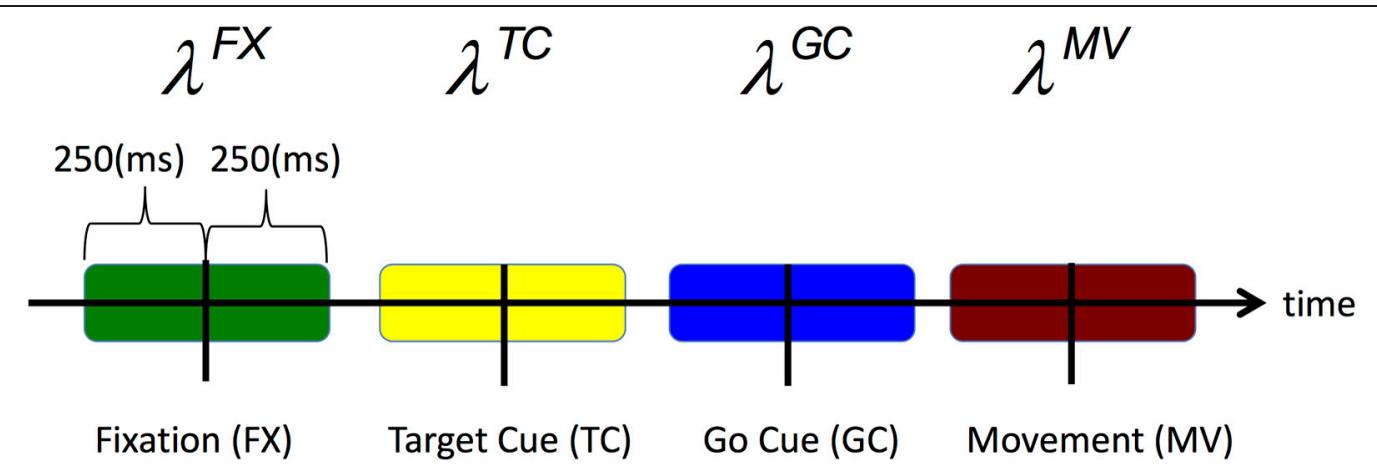

FIGURE 2 | Time periods over which the CIF denoted by Equation (3), is estimated are shaded.

Our model of the stimulus effect, which depends on the movement direction, is

$$
\log \lambda^{S}(t \mid d, \alpha)=\alpha_{d}
$$

The $\alpha=\left\{\alpha_{d}\right\}_{d=1}^{4}$ parameters measure the effects of movement direction on the spiking probability. Our convention is $d=\{1,2,3,4\}=\{\mathrm{Up}$, Right, Down, Left $\}$. For example, if $e^{\alpha_{1}}$ is significantly larger than $e^{\alpha_{2}}, e^{\alpha_{3}}$, and $e^{\alpha_{4}}$ during movement, then the probability that a neuron will spike $(\lambda \cdot \Delta)$, in small time interval $\Delta$ is greater when the patient moves in the "Up" direction, suggesting that the neuron may be tuned in the "Up" direction.

Our model of the spike history effect is

$$
\log \lambda\left(t \mid H_{t}, \beta, \gamma\right)=\sum_{i=1}^{10} \beta_{i} n_{t-j: t-(j+1)}+\sum_{j=1}^{14} \gamma_{j} n_{t-(10 j+9): t-10 j}
$$

where $n_{a: b}$ is the number of spikes observed in the time interval $(a, b]$ during the epoch.

The $\left\{\beta_{j}\right\}_{j=1}^{10}$ parameters measure the effects of spiking history in the previous $10 \mathrm{~ms}$ and, therefore, can capture refractoriness and/or bursting on the spiking probability in the given epoch. For example, if $e^{\beta_{1}}$ is close to zero for any given epoch, then for any given time $t$, if the neuron had a spike in the previous millisecond then the probability that it will spike again is also close to zero (due to refractory period). Or if $e^{\beta_{5}}$ is significantly larger than 1 , then during fixation and for any time $t$, if the neuron had a spike $5 \mathrm{~ms}$ ago then the probability that it will spike again is modulated up, suggesting bursting.

The $\left\{\gamma_{j}\right\}_{j=1}^{14}$ parameters measure the effects of the spiking history in the previous $10-150 \mathrm{~ms}$ on the spiking probability, which may be associated with not only the neuron's individual spiking activity but also that of its local neural network. For example, if $e^{\gamma_{4}}$ is significantly larger than 1 , then for any time $t$ during fixation if the neuron had one or more spikes between 40 and $50 \mathrm{~ms}$ ago then the probability that it will spike again is modulated up, suggesting $20-25 \mathrm{~Hz}$ oscillations. We describe how we used the model parameters to quantify these spiking characteristics in detail in the Results section.
By combining Equations (3), (4), and (5), we see that the CIF GLM for a given neuron may be written as

$$
\begin{aligned}
\log \lambda\left(t \mid d, H_{t}, \theta\right)= & \alpha_{d}+\sum_{i=1}^{10} \beta_{i} n_{t-j: t-(j+1)} \\
& +\sum_{j=1}^{14} \gamma_{j} n_{t-(10 j+9): t-10 j}
\end{aligned}
$$

The model parameter vector $\theta=\{\alpha, \beta, \gamma\}$ contains 28 unknown parameters (for each epoch and for each time window modeled). We computed maximum-likelihood estimates for $\theta$ and $95 \%$ confidence intervals of $\theta$ for each neuron using glmfit.m in MATLAB (Brown et al., 2003). We also used the KolmogorovSmirov (KS) statistic, based on the time-rescaling theorem, to assess model goodness-of-fit (Brown et al., 2002; Truccolo et al., 2005).

Finally, it is important to note that building a point process model of spiking activity of a neuron is equivalent to estimating the joint distribution function for the random spiking process (Sarma et al., 2010). If the estimate of this distribution is satisfactory, then any first and second order statistic (e.g., inter-spike interval histogram, spectrogram, tuning vector, etc.) can be computed using simulated data from the estimated distribution. That is, the point process model encompasses any traditional statistic used to analyze bursting, oscillations or DT in spiking data. Furthermore, traditional statistics can lead to erroneous inferences as shown in more detail in (Sarma et al., 2010).

\section{DETERMINING SPIKE TRAIN CHARACTERISTICS FROM POINT PROCESS MODELS}

Recall from (2) that the product of the rate function for a given neuron and a small time interval, $\lambda\left(t \mid H_{t}, \theta\right) \cdot \Delta$, is approximately the probability that the neuron will fire in time interval $(t, t+\Delta]$ given history of extrinsic and intrinsic dynamics up to time $t$, which is captured in $H_{t}$. Then by virtue of Equations (2) and (6), we allow the probability that each STN neuron will spike at some time $t$ within an epoch (ep) to be modulated by movement direction (captured in $\left\{\alpha_{d}^{e p}\right\}_{d=1}^{4}$ parameters), short-term 
history spiking dynamics (captured in $\left\{\beta_{j}^{e p}\right\}_{j=1}^{10}$ parameters) and long-term history spiking dynamics (captured in $\left\{\gamma_{k}^{e p}\right\}_{k=1}^{14}$ parameters).

Figure 3 shows an example of a single neuron's optimal model parameters and their 95\% confidence intervals during the perimovement epoch. We highlight in Figure 3 and discuss below how certain parameter value ranges indicate refractoriness, bursting, OSCs, and DT.

1. Refractoriness: As illustrated in the second row of Figure 3, the PD STN neuron exhibits refractory periods as indicated by down modulation by a factor of 10 or more due to a spike occurring $1 \mathrm{~ms}$ prior to a given time $t$. That is, if a spike occurs $1 \mathrm{~ms}$ prior to time $t$, then it is very unlikely that another spike will occur at time $t\left(e^{\beta_{1}} \leq 0.1\right.$ for all $e^{\beta_{1}}$ within its $95 \%$ confidence band). Refractoriness is expected since after an action potential (a spike) occurs, as some time (refractory period) must elapse before a neuron can again produce another action potential in response to a stimulus (Brodal, 1998).

2. Bursting: As illustrated in the second row of Figure 3, the PD STN neuron fires in rapid succession before and after movement onset as indicated by one or more of the short-term history parameters $\left(e^{\beta_{i}}\right.$ for $\left.i=2,3, \ldots, 10\right)$ corresponding to $2-10 \mathrm{~ms}$ in the past being larger than 1 . That is, if a spike occurs $2-10 \mathrm{~ms}$ prior to time $t$, then it is more likely that another spike will occur at time $t$. More formally, define $L B_{i}$ and $U B_{i}$ as the $95 \%$ lower and upper confidence bounds for $e^{\beta_{i}}$ such that $L B_{i} \leq e^{\beta_{i}} \leq U B_{i}$ for $i=2,3, \ldots, 10$. Then, if $L B_{i}>1$ and $U B_{i}>1.5$ for at least one $i=2,3, \ldots, 10$, the neuron exhibits bursting.

3. $10-30 ~ H z$ Oscillations (beta): As illustrated in the third row of Figure 3, the PD STN neuron exhibits $10-30 \mathrm{~Hz}$ oscillatory firing before movement. That is, the probability that the PD STN neuron will fire at a given time $t$ is modulated up if a

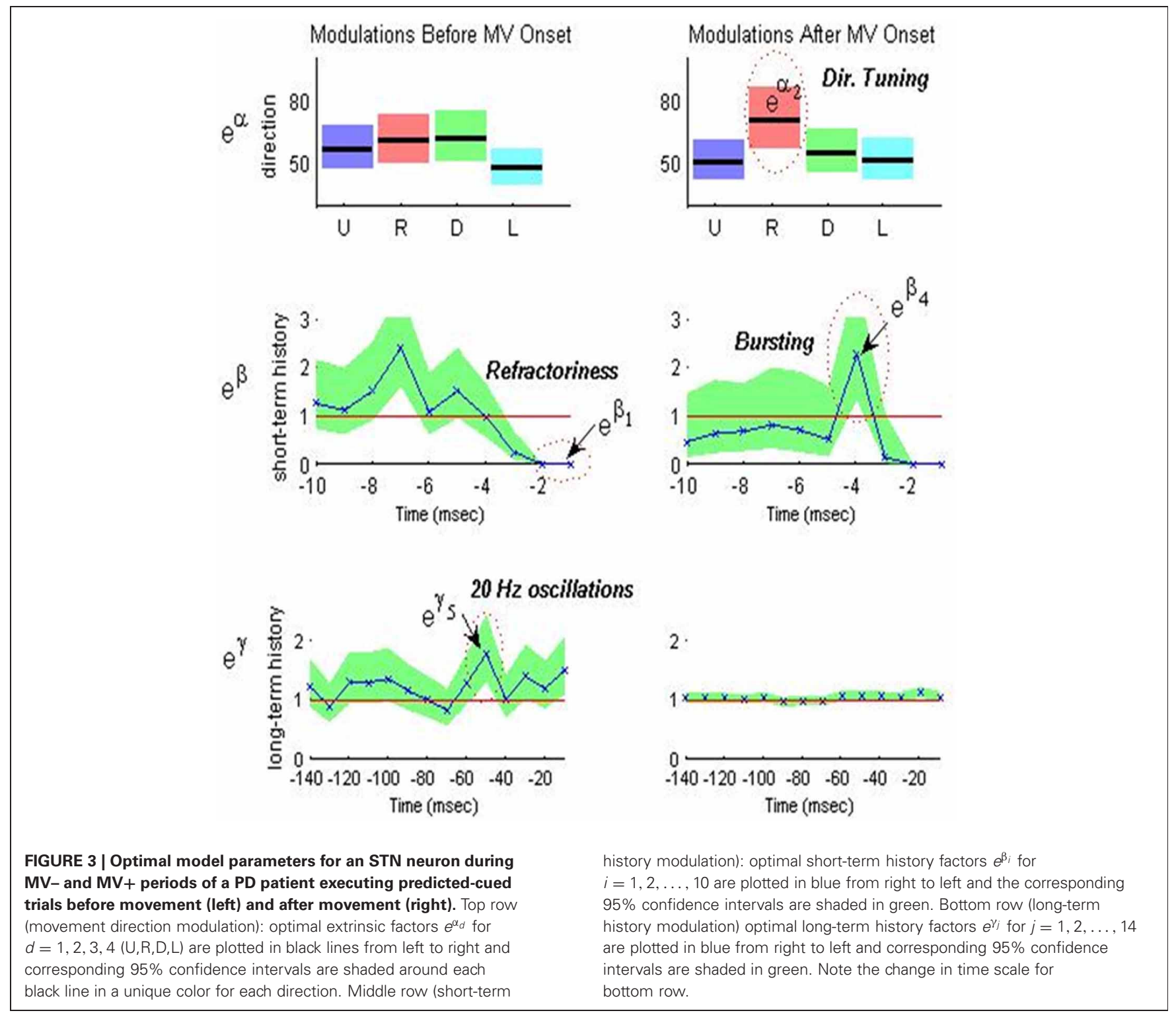


spike occurs 30-100 ms prior to $t$. Again, define $L B_{j}$ and $U B_{j}$ as the $95 \%$ lower and upper confidence bounds for $e^{\gamma_{j}}$ such that $L B_{j} \leq e^{\gamma_{j}} \leq U B_{j}$ for $j=3,4,5$. Then, if $L B_{j}>1$ and $U B_{j}>$ 1.5 for at least one $j=3,4,5$, the neuron exhibits $10-30 \mathrm{~Hz}$ oscillations.

4. Directional Tuning: As illustrated in the first row of Figure 3, the PD STN neuron appears to exhibit more DT after movement onset. That is, the PD neuron seems more likely to fire in one direction more than at least one other direction. To quantify DT, we performed the following test for each neuron and each epoch:

a. For each direction $d^{*}=\{U, R, D, L\}$, compute $\mathrm{p}_{\mathrm{d}^{*}, \mathrm{~d}}=$ $\operatorname{Prob}\left(e^{\alpha_{d^{*}}}>e^{\alpha_{d}}\right)=\operatorname{Prob}\left(\alpha_{d^{*}}>\alpha_{d}\right)$ for $d \neq d^{*}$. Define $p_{\mathrm{d}^{*}, \mathrm{~d}^{*}}=0$. Use the Gaussian approximation for $\alpha_{d}$, which is one of the asymptotic properties of ML estimates to compute $\mathrm{p}_{\mathrm{d}^{*}, \mathrm{~d}}$ (Brown et al., 2003).

b. If $\max _{d^{*}=1,2,3,4} \mathrm{p}_{\mathrm{d}^{*}, \mathrm{~d}} \geq 0.975$ then neuron exhibits DT.

Figure 4 below is a snapshots of the CIF estimate from one STN neuron in a PD patient before movement and after movement along with the spike train data. As shown in the top row of Figure 4, the beta oscillations can be seen in the estimate of the CIF itself as the time between the large amplitude peaks are about $40 \mathrm{~ms}$ apart which corresponds to a $25 \mathrm{~Hz}$ oscillation. These large peaks are attenuated after movement onset (denoted as $t=0$ in Figure 4).

\section{RESULTS}

\section{EFFECTS OF PREDICTABLE AND UNPREDICTABLE CUES ON STN ACTIVITY}

Since we had spike train data for 37 STN neurons across the seven patients (see Table 1), we built point process models for all 37 STN neurons. A total of 28 neuron models passed our goodnessof-fit criterion which required the KS statistic to be within its 95\% confidence bounds (Johnson and Kotz, 1970). Using these 28 models, we determined for each neuron and for each epoch within the trial, whether the neuron exhibited refractoriness, bursting, OSCs, and/or DT.
Figure 5 illustrates a population summary of modulations in bursting, $10-30 \mathrm{~Hz}$ oscillations, and DT for each trial type. We do not plot a summary for refractoriness as $100 \%$ of the neurons exhibited refractoriness during all epochs in all 3 trial types. When the fractional change from baseline (defined to be the first $500 \mathrm{~ms}$ of each trial-fixation or FX) is statistically significant with at least $90 \%$ confidence in a less pathological direction (i.e., decreased bursting, decreased OSC, increased DT), we denote it with a "+" symbol and also note the $p$-value. We used standard sign test to look for significant differences from baseline within each trial type because it is a robust test that does not make any assumptions on the distributions of the random variables we are trying to compare (Zar, 1999). In this case, the two random variables we compare for each epoch after fixation within a trial are (1) the percentage of neurons that exhibit a characteristic during the epoch (2) the percentage of neurons that exhibit a characteristic during fixation.

As shown in Figure 5, during predicted + cue trials (top row), there is an increase in DT and a decrease in beta oscillations early on during the trial immediately after target cue onset. After movement, this suppression of pathological activity becomes more pronounced, which has been previously reported in studies where patients could predict go cues (Alexander and Crutcher, 1990; Amirnovin et al., 2004; Williams et al., 2005; Gale et al., 2009; Sarma et al., 2010). During unpredicted trials where the absence of a cue triggers movement (Figure 5, bottom row), we also see an increase in DT and a decrease in beta oscillations later on during the trial (on average $640 \mathrm{~ms}$ after target cue onset).

Interestingly, during unpredicted + cue trials (middle row), we did not observe significant suppression of beta oscillations or significant increase in DT at any time during the trial even though cues were presented.

\section{EFFECTS OF PREDICTABLE AND UNPREDICTABLE CUES ON BEHAVIOR}

Distributions of behavior for each trial type are given in Figure 6. The average reaction time for predicted +cue trials is $0.69 \mathrm{~s}$, and the average movement times for predicted +cue trials and

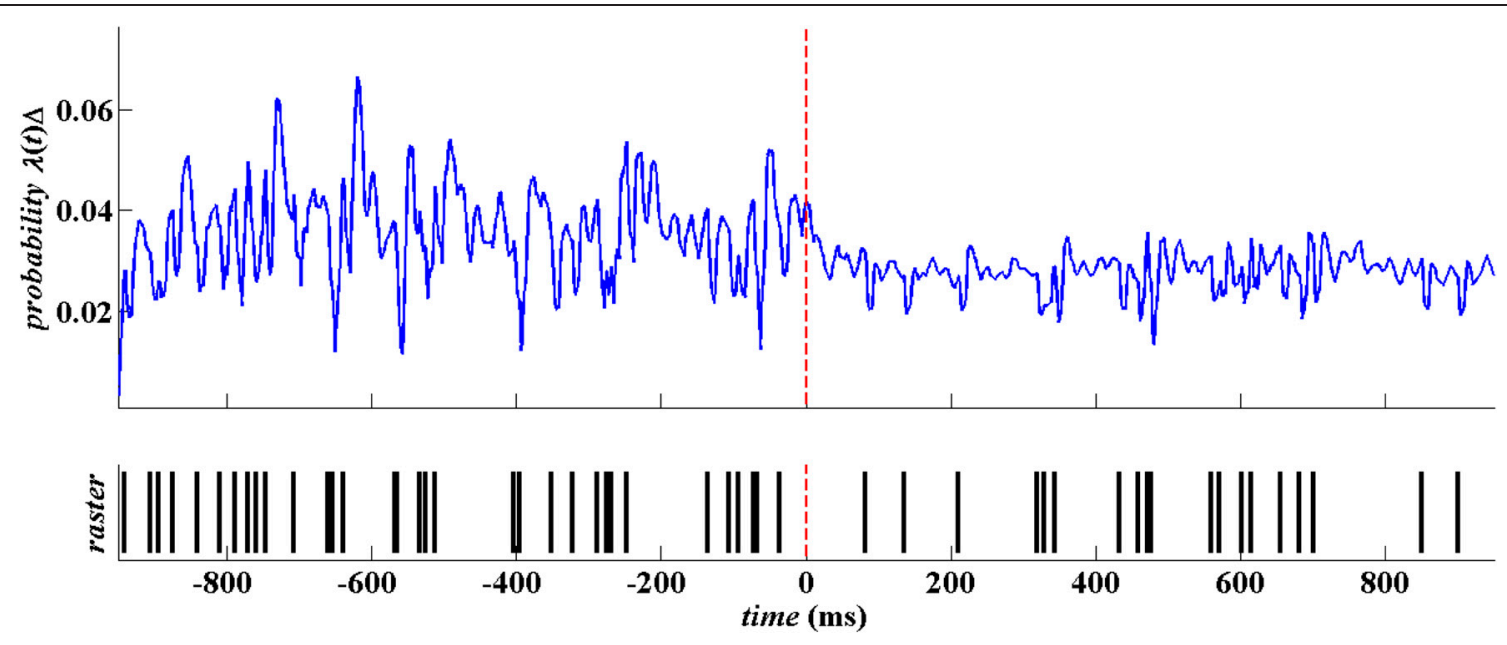

FIGURE 4 | Raw spike train data from a single STN neuron in PD patient. The spike train is in blue and the corresponding estimate of the CIF is in red. 


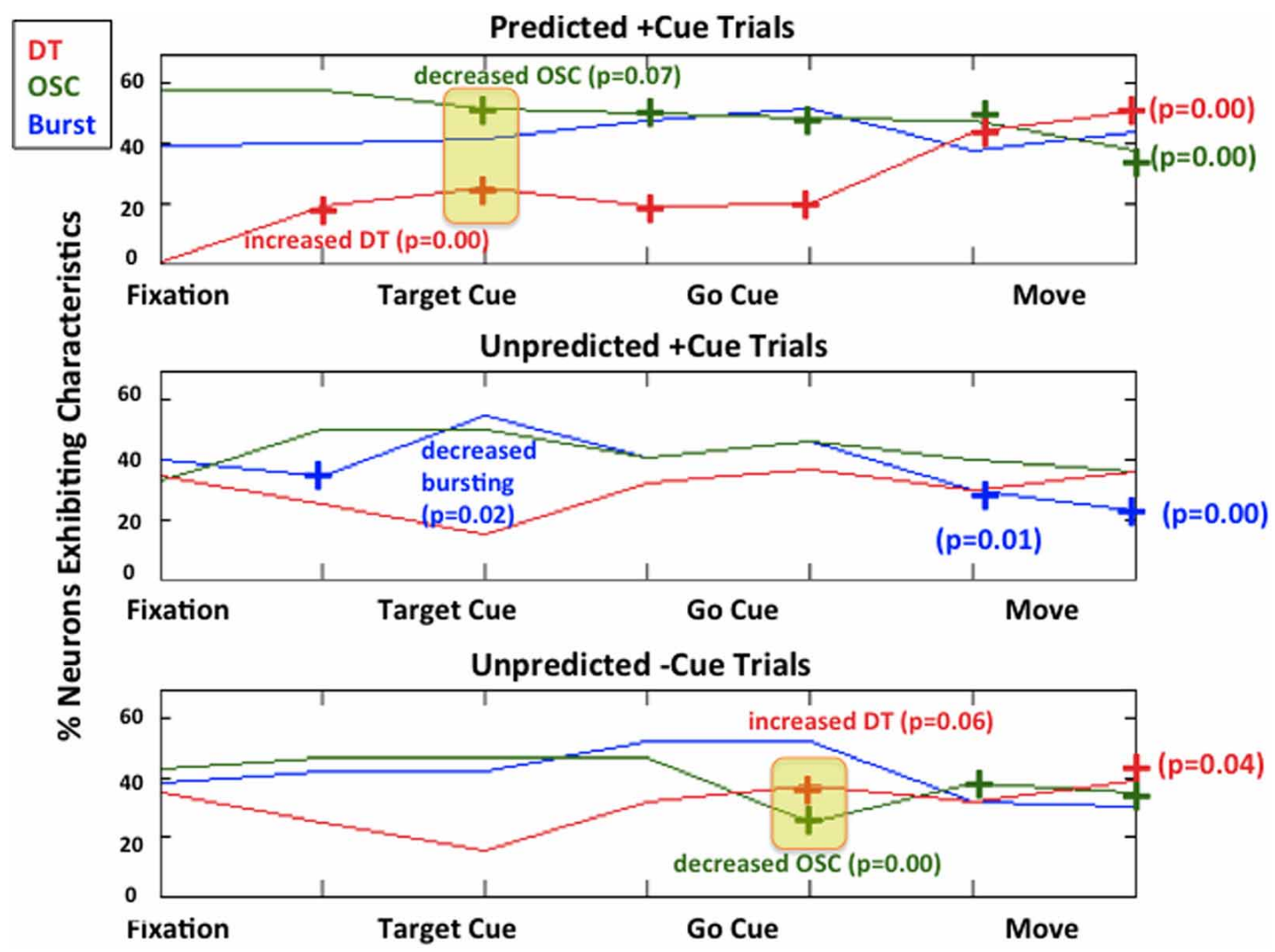

FIGURE 5 | Modulations of each characteristic for each trial type.

Predicted +Cue Trials (top); Unpredicted +Cue Trials (middle);

Unpredicted -Cue Trials (bottom). When the percentage of neurons exhibit neuronal spiking characteristics in a monotonically decreasing less pathological direction (decreasing beta oscillations, increasing directional tuning) for the duration of the trial, we denote that with a "+" symbol.

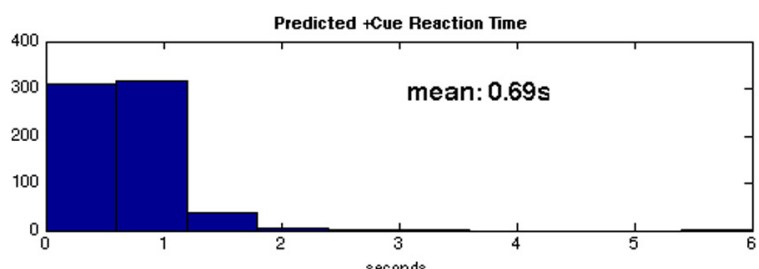

UnPredicted +Cue Reaction Time

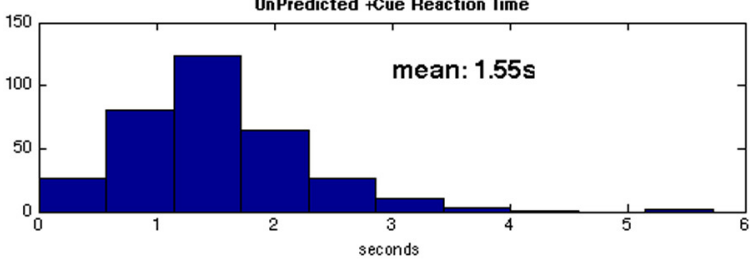

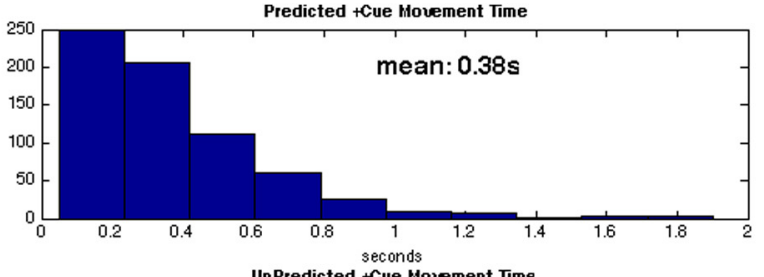
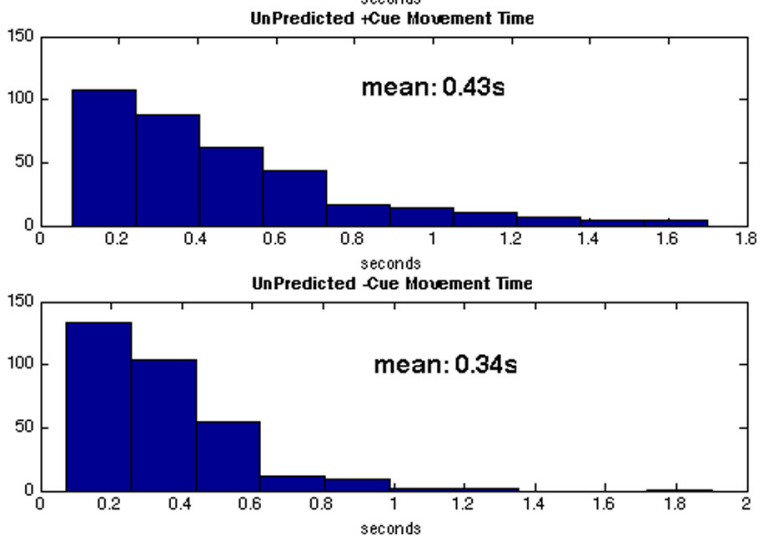

FIGURE 6 | Distributions for reaction times (left) and movement times (right) for each trial type. 
self-initiated -cue trials are 0.38 and $0.34 \mathrm{~s}$, respectively. On the other hand, motor performance deteriorated in the unpredicted + cue trials. Specifically, the average movement and reaction times are 0.43 and $1.55 \mathrm{~s}$, respectively.

For behavioral responses, we tested for statistically significant differences between the 3 trial types using a series of twosample KS test (Johnson and Kotz, 1970). The two-sample KS test checks whether the two data samples come from the same distribution and does not specify what that common distribution is (e.g., normal or not normal). We summarize the results in Table 2.

As shown in Table 2, the predicted + cue trials and unpredicted -cue trials do not significantly differ in movement times. In contrast, the predicted + cue trials and the unpredicted +cue trials significantly differ in both movement and reaction times. The unpredicted + cue and unpredicted - cue trials also significantly differ in movement times. These results suggest that behavioral performance is comparable only during predicted +cue and unpredicted-cue trials, which are the only two trial types for which we also observe suppression of pathological neural activity.

\section{DISCUSSION}

To our knowledge, previous works that study the effects of cues on BG neurophysiology in PD patients entail experimental set ups for which cues can be entirely predicted by patients. Two important questions that we ask here, which may shed new light on the underlying mechanisms behind cue-related movements, are (1) "what happens when the timing of cues cannot be predicted by patients?" and (2) "what if an expected cue never appears forcing the patient to move in the absence of a cue?" Specifically, we took a traditional directed hand-movement task and split it into cases where cues can and cannot be predicted before the start of each trial. Our hypothesis was that the inability to predict the timing of a presented external cue would diminish dampening of pathological BG activity observed with cue presentation when cue timing is predictable.

\section{EFFECTS OF TIMING OF EXTERNAL CUES}

Two of the trial types performed, predicted + cue and unpredicted $+c u e$, were identical in terms of visuospatial timing and presentation, including the presence of go cues in both (top and middle, Figure 1). The only difference between these two task conditions was the subject's ability to predict the timing of the go cue. This anticipatory difference resulted in increasing suppression of

Table 2 | Results for 2 sample KS test for all possible trial pair comparisons.

\begin{tabular}{lll}
\hline Two trials to compare & Behavior variable & 2 sample KS test results \\
\hline Predicted +cue & Reaction times & Reject Null Hypothesis \\
Unpredicted +cue & & $p$-value $=3.63 \times 10^{-64}$ \\
Predicted +cue & Movement times & Reject Null Hypothesis \\
Unpredicted +cue & & $p$-value $=2.72 \times 10^{-6}$ \\
Predicted +cue & Movement times & Accept Null Hypothesis \\
Unpredicted +cue & & $p$-value $=0.1007$ \\
Unpredicted +cue & Movement times & Reject Null Hypothesis \\
Unpredicted +cue & & $p$-value $=0.0052$
\end{tabular}

pathological beta oscillations beginning early on during the trial and improvement in reaction time and movement time in the former compared to the latter.

These results suggest that the timing of the external cue must be anticipated to activate a motor plan and effectively trigger movement in PD. There is evidence that premotor cortical areas show increased activity when the timing of an external cue is predictable in patients (Jahanshahi et al., 1995; Paradiso et al., 2003). Since there are direct projections (hyperdirect pathway) to the STN from these cortical areas (Carpenter et al., 1981; Canteras et al., 1990), the firing rates in the STN also show increased activity from baseline (Paradiso et al., 2003). Consequently, the pathological beta oscillations in STN seen in PD may be dampened, perhaps by inactivation of the resurgent sodium current (Do and Bean, 2003). Finally, dampening of these excessive oscillations may facilitate movement.

\section{EFFECTS OF INTERNAL CUES GENERATED BY THE ABSENCE OF EXPECTED EXTERNAL CUES}

In contrast, two of the trial types, the predicted + cue and unpredicted-cue conditions, resulted in suppression of pathological beta oscillations and improvement in behavioral measures. As we noted, the timing of the suppression occurred later in the unpredicted -cue trial type. The suppression of pathological BG oscillations occurred in both trial types despite their visuospatial dissimilarity, with the presence of the go cue in the former and the lack of a go cue in the latter.

In the unpredicted - cue condition, our PD subjects were compelled to move by an impending deadline, and movements were triggered in the absence of a cue. There is a $50 \%$ chance the external go cue will appear at the start of each trial. If the subject does not move by the end of the go cue epoch, which is defined whether the cue is presented or not, the subject fails the trial and no reward is received. Thus, at some point during the go cue epoch, the subject decides to self-initiate movement in the absence of an external cue. We term this internal impetus to move an "internally generated" cue. Our findings suggest that this internally generated cue is as effective as the external cue in the predicted condition in continuously suppressing beta oscillations, increasing DT, and decreasing movement time.

These findings are consistent with those reported in (Jahanshahi et al., 1995) which also showed that premotor cortical areas show increased activity when movements are triggered internally (e.g., self-initiated movements). Therefore, the internal impetus to move may activate prefrontal cortical activity that then triggers the same downstream effects that dampen pathological activity and facilitate movement as do predicted external cues, without requiring the presentation of the external cue.

Although there is no way to determine when the internal cue was generated by the subject, the internal cue should be generated on average after the external go cue would usually have appeared; that is, when the subject realizes that the external cue is not coming and an internal cue is necessary. This leads to the prediction that, if both the internal and the external cues result in PD movement facilitation via the same physiological mechanism, this modulation would occur earlier in predicted +cue trials than in unpredicted - cue trials. Indeed, we find the neurophysiological changes seen in beta oscillations and DT occur on average $640 \mathrm{~ms}$ 
after those seen in predicted +cue trials, as we would have predicted.

Why does the unpredicted +cue condition result in greater oscillatory activity in the beta band, decreased DT after movement onset, and increased reaction and movement times in comparison to the other two conditions? An "expectation of movement" may be important in both clinical PD behavior and the physiology of a cue-related response. It is well known that cues activate the PD condition. The use of different cue types in assistive devices to augment the activation required for motor movements is believed to function by creating such an expectation of movement, decreasing beta power in the BG and motor cortex prior to movement onset.

To study this phenomenon, we had created a task that creates both expected and unexpected cue conditions. In the expected cue condition, we hypothesized and found a decrement in beta oscillatory activity after cue presentation, before movement onset. This is in keeping with the idea that an "expectation of movement" is required prior to movement onset, resulting in decreased beta activity in preparation for movement. In the unexpected -cue condition, we see that the decrement in beta power occurs later, in keeping with our prediction that there would be a lag associated with the self-generation of an internal cue to move. Once again, the internally generated cue sets in motion the preparatory decrease in beta activity prior to movement.

Our most interesting finding, however, is seen in the unexpected +cue condition. One may reasonably expect that in the presence of a cue, there would be a decrement in beta whether the cue is expected or unexpected. That is not what we hypothesized or found. Instead, beta remains present to a greater degree than in the expected +cue and the unexpected - cue conditions, after cue presentation. This is accompanied by greater movement time, as if the effort to move is handicapped by the unexpected nature of the cue. So what is going on?

We believe that the unexpected nature of the cue, in a PD environment, prevents the cell from adequately preparing for movement. We know that prior to movement onset, beta power in a normal subject decreases in the BG as well as motor cortex. This diminishment of beta in preparation for movement requires that the movement is anticipated. The lack of anticipatory capability in our experimental "unexpected +cue" condition prevents the beta decrease that necessarily precedes movement, while at the same time giving the system the "go cue" to move while the system is in an unprepared state. Without the ability to anticipate, in the "unexpected +cue" condition the preparatory decrease in beta does not occur normally.

Yet we have another unexpected condition, -cue, where beta did diminish appropriately prior to movement. The unexpected - cue condition differs from the unexpected +cue condition, however. Here, the expectation of movement was internally generated and movement preparation was carried out, thus creating a time lag to movement not seen in the "unexpected +cue" condition. Without a go cue, the system was free to first prepare for movement, and then generate an internal go cue that triggered movement that followed movement preparation. However when presented with an unexpected external go cue, no preparatory phase was possible. The movement in response to the go cue was, therefore, made hesitantly and haltingly, with beta activity present and movement time lengthening as a result of the lack of movement preparation.

What is the significance of this finding? We believe that we have found an experimental test condition that mimics the condition found in PD. In the Parkinsonian condition, there is an excess of beta oscillatory power. This abnormality is seen both in BG and motor cortex (Hutchison et al., 1994; Marsden et al., 2001; Brown, 2003; Gale et al., 2008; Hammond et al., 2007; Sarma et al., 2010). Movement in the PD patient occurs in the presence of greater beta than in normal subjects in STN and motor cortex. Our task strategy presented for the subject an unexpected cue for movement, providing no time for preparatory beta decrease. In a normal subject, our experimental condition may mimic PD by providing an elevated beta at the time of movement onset that would not be seen in a more typical scenario where environmental cues are expected.

However, these experiments were performed with Parkinsonian patients with existing abnormal beta dynamics. As a result, we see a worsening of existing beta that is additive to the already elevated beta seen in PD. Movement times are longer in PD patients, and in the "unexpected + cue" condition movement time was further extended. It would be interesting to see, outside the PD state in a normal subject, whether there would be excess beta after an unexpected cue. Stated alternatively, it is entirely possible that our ability to see this phenomenon results from abnormalities in movement preparation that is present only in the PD condition due to its abnormal PD dynamics.

What may be critical for motor facilitation in PD is a clear trigger that activates a pre-existing motor plan already formulated in prefrontal cortex. The mechanism of this activation would require the "expectation of movement" in the form of an expected external cue, or it can also be an internally generated cue (in our case generated in the absence of a visual cue) to move. In future work, we will record movement-related potentials for the same two-step task while we also record single unit neuronal activity in the STN to test the following hypothesis: it is the activation of a specific motor plan, not the necessarily presentation of a cue, which is the critical event that provides the cortical drive that modulates the abnormal physiology of the basal ganglia, leading to motor facilitation in Parkinson's disease. The importance here is that movement facilitation in PD does not derive from generalized cortical activation, or the activation of sensory cortical circuitry at any primary or associative level. This adds significant nuance to the neurophysiology of PD dysfunction that would be critical in future discussion of the disease. It would also be critical in devising potential future stimulation therapies, for example, that may be based upon finely tuned cortical activation.

\section{ACKNOWLEDGMENTS}

This work is supported by Burroughs Wellcome Fund CASI Award 1007274, the National Science Foundation CAREER Award 1055560, and NIH R01NS073118-02 to Sridevi V. Sarma; National Institute on Drug Abuse (DA015644 and MH59733 to Emery N. Brown); Neurosurgery Research Education Foundation; Parkinson Disease Foundation to Emad Eskandar; and Neurosurgery Research Education Foundation to Ming L. Cheng. 


\section{REFERENCES}

Abosch, A., Hutchison, W. D., SaintCyr, J. A., Dostrovsky, J. O., and Lozano, A. M. (2002). Movement related neurons of the subthalamic nucleus in patients with Parkinson disease. J. Neurosurg. 97, 1167-1172.

Alexander, G. E., and Crutcher, M. D. (1990). Preparation for movement: neural representations of intended direction in three motor areas of the monkey. J. Neurophysiol. 64, 133-150.

Amirnovin, R., Williams, Z. M., Cosgrove, G. R., and Eskandar, E. N. (2004). Visually guided movements suppress subthalamic oscillations in Parkinson's disease patients. J. Neurosci. 24, 11302-11306.

Amirnovin, R., Williams, Z. M., Cosgrove, G. R., and Eskandar, E. N. (2006). Experience with microelectrode guided subthalamic nucleus deep brain stimulation. Neurosurgery 58(Suppl. 1), ONS96-ONS102.

Azulay, J. P., Mesure, S., Amblard, B., Blin, O., Sangla, I., and Pouget, J. (1999). Visual control of locomotion in Parkinson's disease. Brain 122, 111-120.

Azulay, J. P., Van Den Brand, C., Mestre, D., Blin, O., Sangla, I., Pouget, J., and Serratrice, G. (1996). Automatic motion analysis of gait in patients with Parkinson's disease: effects of levodopa and visual stimulations. Rev. Neurol. (Paris) 152, 128-134.

Barbieri, R., Quirk, M. C., Frank, L. M., Wilson, M. A., and Brown, E. N. (2001). Construction and analysis of non-Poisson stimulus response models of neural spike train activity. J. Neurosci. Methods 105, 25-37.

Bergman, H., Wichman, T., Karmon, B., and DeLong, M. R. (1994). The primate subthalamic nucleus. II. Neuronal activity in the MPTP model of parkinsonism. J. Neurophysiol. 72, 507-520.

Brodal, P. (1998). The Central Nervous System: Structure and Function. New York, NY: Oxford University Press.

Brown, P. (2003). Oscillatory nature of human basal ganglia activity: relationship to the pathophysiology of Parkinson's disease. Mov. Disord. 357-363.

Brown, E. N., Barbieri, R., Eden, U. T., and Frank, L. M. (2003). "Likelihood methods for neural data analysis," in Computational Neuroscience: A Comprehensive Approach, ed J. Feng (London: CRC), 253-286.

Brown, E. N., Barbieri, R., Ventura, V., Kass, R. E., and Frank, L. M. (2002). The time-rescaling theorem and its application to neural spike train data analysis. Neural Comput. 14, 325-346.

Brown, E. N., Nguyen, D. P., Frank, L. M., Wilson, M. A., and Solo, V. (2001a). An analysis of neural receptive field dynamics by point process adaptive filtering. Proc. Natl. Acad. Sci. U.S.A. 98, 12261-12266.

Brown, P., Oliviero, A., Mazzone, P., Insola, A., Tonali, P., and Lazzaro, V. D. (2001b). Dopamine dependency of oscillations between subthalamic nucleus and pallidum in Parkinson's disease. J. Neurosci. 21, 1033-1038.

Canteras, N. S., Shammah-Lagnado, S. J., Silva, B. A., and Ricardo, J. A. (1990). Afferent connections of the subthalamic nucleus: a combined retrograde and anterograde horseradish peroxidase study in the rat. Brain Res. 513, 43-59.

Carpenter, M. B., Carleton, S. C., Keller, J. T., and Conte, P. (1981) Connections of the subthalamic nucleus in the monkey. Brain Res. 224, 1-29.

Crutcher, M. D., and DeLong, M. R (1984). Single cell studies of the primate putamen. II. Relations to direction of movement and pattern of muscular activity. Exp. Brain Res. 53, 244-258.

Czanner, G. C., Eden, U. T., Wirth, S. Yanike, M., Suzuki, W., and Brown, E. N. (2008). Analysis of betweentrial and within-trial neural spiking dynamics. J. Neurophysiol. 99, 2672-2693.

Daley, D., and Vere-Jones, D. (2003). An Introduction to the Theory of Point Process, 2nd edn. New York, NY: Springer-Verlag.

Do, M. T., and Bean, B. P. (2003) Subthreshold sodium currents and pacemaking of subthalamic neurons: modulation by slow inactivation. Neuron 39, 109-120.

Dostrovsky, J., and Bergman, H. (2004). Oscillatory activity in the basal ganglia-relationship to normal physiology and pathophysiology. Brain 127, 721-722.

Eden, U. T., Amirnovin, R., Brown, E. N., and Eskandar, E. N. (2007) "Constructing models of the spiking activity of neurons in the subthalamic nucleus of Parkinson's patients," in Proceedings of Joint Statistical Meetings (JSM), (Salt Lake City, UT).

Gale, J. T., Amirnovin, R., Williams, Z. M., Flaherty, A. W., and Eskandar, E. N. (2008). From symphony to cacophony: pathophysiology of the human basal ganglia in Parkinson disease. Neurosci. Biobehav. Rev. 32, 378-387.
Gale, J. T., Shields, D. C., Jain, F. A., Amirnovin, R., and Eskandar, E. N. (2009). Subthalamic nucleus discharge patterns during movement in the normal monkey and Parkinsonian patient. Brain Res. 1260, 15-23.

Georgiou, N., Iansek, R., Bradshaw, J. L., Phillips, J. G., Mattingley, J. B. and Bradshaw, J. A. (1993). An evaluation of the role of internal cues in the pathogenesis of Parkinsonian hypokinesia. Brain 116, 1575-1587.

Glickstein, M., and Stein, J. (1991) Paradoxical movement in Parkinson's disease. Trends Neurosci. 14, 480-482.

Hammond, C., Bergman, H., and Brown, P. (2007). Pathological synchronization in Parkinson's disease: networks, models and treatments. Trends Neurosci. 30, 357-364.

Hutchison, W. D., Allan, R. J., Opitz, H., Levy, R., Dostrovsky, J. O., Lang, A. E., and Lozano, A. M. (1998). Neurophysiological identification of the subthalamic nucleus in surgery for Parkinson's disease. Ann. Neurol. $44,622-628$.

Hutchison, W. D., Dostrovsky, J. O. Walters, J. R., Courtemanche, R., Boraud, T., Goldberg, J., and Brown, P. (1994). Neuronal oscillations in the basal ganglia and movement disorders: evidence from whole animal and human recordings. J. Neurosci. 24, 9240-9243.

Jahanshahi, M., Jenkins, I. H., Brown, R. G., Marsden, C. D., Passingham, R. E., and Brooks, D. J. (1995). Self-initiated versus externally triggered movements. I. An investigation using measurement of regional cerebral blood flow with PET and movement-related potentials in normal and Parkinson's disease subjects. Brain 119(Pt 3), 1045-1048.

Johnson, A., and Kotz, S. (1970). Distributions in Statistics: Continuou Univariate Distributions, New York, NY: Wiley.

Kass, R. E., and Ventura, V. (2001). A spike train probability model. Neural Comput. 13, 1713-1720.

Keat, J., Reinagel, P., Reid, R. C., and Meister, M. (2001). Predicting every spike: a model for the responses of visual neurons. Neuron 30 , 803-817.

Kompoliti, K., Goetz, C. G., Leurgans, S., Morrissey, M., and Siegel, I. M. (2000). On freezing in Parkinson's disease: resistance to visual cue walking devices. Mov. Disord. 15, 309-312.

Kühn, A. A., Williams, D., Kupsch, A., Limousin, P., Hariz, M., Schneider, G. H., Yarrow, K., and Brown, P.
(2004). Event-related beta desynchronization in human subthalamic nucleus correlates with motor performance. Brain 127(Pt 4), 721-722.

Lang, A. E., and Lozano, A. M. (1998a). Parkinson's disease. First of two parts. N. Engl. J. Med. 339, 1044-1053.

Lang, A. E., and Lozano, A. M. (1998b). Parkinson's disease. Second of two parts. N. Engl. J. Med. 339, 1130-1143.

Levy, R., Ashby, P., Hutchison, W. D., Lang, A. E., Lozano, A. M. and Dostrovsky, J. O. (2002). Dependence of subthalamic nucleus oscillations on movement and dopamine in Parkinson's disease. Brain 125, 1196-1209.

Levy, R., Dostrovsky, J. O., Lang, A. E., Sime, E., Hutchison, W. D., and Lozano, A. M. (2001). Effects of apomorphine on subthalamic nucleus and globus pallidus internus neurons in patients with Parkinson's disease. J. Neurophysiol. 86, 249-260.

Levy, R., Hutchison, W. D., and Lozano, A. M., and Dostrovsky, J. O. (2000). High-frequency synchronization of neuronal activity in the subthalamic nucleus of Parkinsonian patients with limb tremor. J. Neurosci. 20, 7766-7775.

Majsak, M. J., Kaminski, T., Gentile, A. M., and Flanagan, J. R. (1998). The reaching movements of patients with Parkinson's disease under selfdetermined maximal speed and visually cued conditions. Brain 121, 755-766.

Marsden, J. F., Limousin-Dowsey, P., Ashby, P., Pollak, P., and Brown, P. (2001). Subthalamic nucleus, sensorimotor cortex and muscle interrelationships in Parkinson's disease. Brain 124, 378-388.

McCullagh, P., and Nelder, J. A. (1989) Generalized Linear Models, 2nd edn. Boca Raton, FL: Chapman and Hall/CRC.

Montgomery, E. Jr. (2008). Subthalamic nucleus neuronal activity in Parkinson's disease and epilepsy subjects. Parkinsonism Relat. Disord. 14, 120-125.

Morris, M. E., Lansek, R., Matyas, T. A., and Summers, J. J. (1996). Stride length regulation in Parkinson's disease: normalization strategies and underlying mechanism. Brain 119, 551-568.

Paninski, L. (2004). Maximum likelihood estimation of cascade pointprocess neural encoding models. Network 15, 243-262.

Paradiso, G., Saint-Cyr, J. A., Lozano, A. M., Lang, A. E., and Chen, R. 
(2003). Involvement of the human subthalamic nucleus in movement preparation. Neurology 61, 1538-1545.

Sarma, S. V., Cheng, M., Williams, Z., $\mathrm{Hu}, \mathrm{R} .$, Eskandar, E., and Brown, E. N. (2010). Comparing healthy and Parkinsonian neuronal activity in sub-thalamic nucleus using point process models. IEEE Trans. Biomed. Eng. 57, PMCID20172804.

Snyder, D. L., and Miller, M. I. (1991). Random Point Processes in Time and Space. New York, NY: Springer.

Suteerawattananon, M. (2004). Effects of visual and auditory cues on gait in individuals with Parkinson's disease. J. Neurol. Sci. 219, 63-69.
Truccolo, W., Eden, U. T., Fellow, M. R., Donoghue, J. P., and Brown, E. N. (2005). A point process framework for relating neuronal spiking activity for spiking history, neural ensemble and extrinsic covariate effects. J. Neurophysiol. 93, 1074-1089.

Truccolo, W., Hochberg, L. R., and Donoghue, J. P. (2010). Collective dynamics in human and monkey sensorimotor cortex: predicting single neuron spikes. Nat. Neurosci. 13, 105-111.

Williams, Z. M., Neimat, J. S., Cosgrove, G. R., and Eskandar, E. N. (2005). Timing and direction tuning of subthalamic and pallidal neurons in patients with
Parkinson disease. Exp. Brain Res. 162, 407-416.

Zar, J. H. (1999). Biostatistical Analysis, 4th edn. Upper Saddle River, NJ: Prentice Hall.

Zelnikera, E., Bradley, A. P., Castnerc, J. E., Cheneryc, H. J., Copland, D. A., and Silburnd, P. A. (2008). Estimation of neuronal firing rates with the three-state biological point process model. J. Neurosci. Methods 174, 281-291.

Conflict of Interest Statement: The authors declare that the research was conducted in the absence of any commercial or financial relationships that could be construed as a potential conflict of interest.
Received: 30 January 2012; accepted: 15 June 2012; published online: 26 July 2012.

Citation: Sarma SV, Cheng ML, Eden U, Williams Z, Brown EN and Eskandar E (2012) The effects of cues on neurons in the basal ganglia in Parkinson's disease. Front. Integr. Neurosci. 6:40. doi: 10.3389/fnint.2012.00040

Copyright (C) 2012 Sarma, Cheng, Eden, Williams, Brown and Eskandar. This is an open-access article distributed under the terms of the Creative Commons Attribution License, which permits use, distribution and reproduction in other forums, provided the original authors and source are credited and subject to any copyright notices concerning any thirdparty graphics etc. 